\title{
Application of a Supervised Improved PID for the scheduling of energy feeding in a PV - battery system
}

\author{
Lucio Ciabattoni * Gianluca Ippoliti * Matteo Cavalletti ** \\ Marco Rocchetti ${ }^{* *}$ Sauro Longhi* \\ * Dipartimento di Ingegneria dell'Informazione, Universita' Politecnica \\ delle Marche, Via Brecce Bianche, 60131 Ancona, Italy \\ (e-mail: \{l.ciabattoni, gianluca.ippoliti, sauro.longhi\}@univpm.it). \\ ** Energy Resources SPA, Via Ignazio Silone, Jesi (AN), 60035 Italy \\ (e-mail: \{marco.rocchetti, matteo.cavalletti\}@energyresources.it)
}

\begin{abstract}
This paper deals with the development of a PID control architecture for better utilization of the storage battery connected to a PhotoVoltaic (PV) Plant. The problem of the stochastic nature of the PV plant is overcomed scheduling the power feeding of the electric line. A neural network is used to derive the one-day-forecast of the PV production and a supervised PID controller is proposed to control the charge and discharge current reference to the battery, that is used as an energy buffer. The communication between all the parts of the system and the supervisor controller is made via TCP/IP protocol. The Energy Resources company has supported the experimentally tests of the proposed solution on a $14 \mathrm{KWp} \mathrm{PV} \mathrm{plant} \mathrm{and} \mathrm{a}$ lithium battery pack.
\end{abstract}

Keywords: Battery, Photovoltaic, Supervised PID, Smart Grid, Renewable Energies.

\section{INTRODUCTION}

As concerns about climate change, rising fossil fuel prices, and energy security increase, there is growing interest around the world in renewable energy resources. Since most renewable energy sources are intermittent in nature, it is a challenging task to integrate a significant portion of renewable energy resources into the power grid infrastructure. Traditional electricity grid was designed to transmit and distribute electricity generated by large conventional power plants. The electricity flow mainly takes place in one direction from the centralized plants to consumers. In contrast to large power plants, renewable energy plants have less capacity, and are installed in a more distributed manner at different locations. The integration of distributed renewable energy generators has great impacts on the operation of the grid and calls for new grid infrastructure. Indeed, it is a main driver to develop the smart grid for infrastructure modernization, which monitors, protects, and optimizes the operation of its interconnected elements from end to end with a two-way flow of electricity and information to create an automated and distributed energy delivery network. On the other hand, while renewable energy systems, such as photovoltaic and wind plants, have problems of controllability due to their intermittent output it becomes necessary to find a way to schedule power output in a deterministic manner. This problem has been deeply investigated in literature, in particular for what regards the forecasting and power scheduling from wind plant (Strunz et al. (2003), Lange et al. (2005)). The prediction of solar yields is becoming more and more important, especially for countries where legislation en- courages the deployment of solar power plants (see Lorenz et al. (2009)). In Italy, where the installed photovoltaic (PV) power amounts is more than $8 \mathrm{GW}$, a proposal of law of 2009 included $20 \%$ more incentives for the correct hourly prediction of the power production of the next day (with an error of less than 10\%) for at least 300 days/year. Forecast information on the expected solar power production is necessary for the management of electricity grids, for scheduling of conventional power plants and also for decision making on the energy market, as described in Lange (2006). There are models including those called clear-day solar radiation, half-sine, Colares-Pereirs and Rabl, and ARIMA hour-by-hour solar irradiance (see Li et al. (1998); Ren et al. (2002); Dorvlo et al. (2002) and reference therein), but they're used to derive irradiation forecast up to one hour. In this work the considered forecasting scheme is based on RBF Neural Network and used to derive predictions of solar irradiation based on forecasts up to 48 hours ahead provided by meteo websites and Energy Resources' database of measured solar irradiation. The irradiation forecast is used as input for a PV's model to obtain the forecast of the PV production. The last element to provide scheduled power output is the storage system, a lithium battery pack used as an energy buffer to store the exceding power produced or to supply the lack of power compared with the reference. To achieve this target is proposed a PID (Proportional, Integral and Differential) controller with a supervisor that can activate some features in order to obtain a globally satisfactory step response and set-point tracking.

The paper is organized in the following way. In Section 2 , some details on the system architecture are recalled, 
the proposed PID algorithm with its improvements are described in Section 3, the PV production forecast method is briefly discussed in Section 4. The implementation settings and the analysis of the control performance are reported in Section 5.

\section{SYSTEM ARCHITECTURE}

Proportional-Integral-Differential (PID) controller is by far the most popular feedback controller in the field of automatic control. Following the lines of literature (Ippoliti et al. (2005), Copeland et al. (1994), Sehab (2007)) the approach proposed here situates in the area of supervisory control, whose greater flexibility and potentiality with respect to fixed controllers is currently being more and more recognized. The motivations for applying supervisor strategies to the design of PID controllers can be outlined as follows. When using a single, time-invariant PID, a non fully satisfactory closed loop performance can be obtained, even if the PID is properly chosen. Large overshoot, poorly damped oscillations and too long rise time need to be avoided in our application, as those situations can seriously damage the devices of the system or compromise the performance distancing the achievement of the aim. To reduce the aforementioned inconveniences, it appears quite natural to design a PID controller which can be modified, from time to time, according to the characteristics of the produced step response (see Liu et al. (2010)). The time varying control law is here realized allowing a supervisor to select the most appropriate among a predefined list of features. The idea is to define an activation policy among some fixed features to obtain a globally satisfactory step response and set-points tracking.

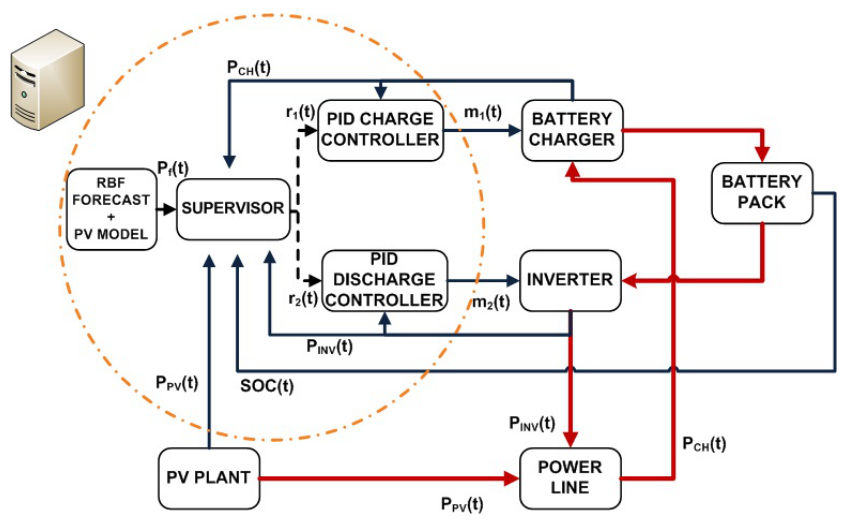

Fig. 1. Scheme of the whole system.

In figure 1 red continuous lines represent power fluxes, blue continuous lines are the information fluxes that goes over TCP/IP protocol and black dotted lines are datas exchanged between software components. The supervisor, the RBF network and PID controllers are Labview softwares located on a server in the base of the company Energy Resources. The aim of the supervised controller is to keep the total instant power feeding the power line (composed by $\mathrm{PV}$ production, inverter generation and power absorbed by the battery charger) equal to the PV production forecast. As appears in fig 1, two different PID controllers regulates the inverter power generation and battery charging. The setpoint $r_{1}(t)$ and $r_{2}(t)$ of PID controllers (respectively the Inverter and Battery Charger one) is computed by the supervisor from the difference $r(t)$ between the forecasted PV production and the measured one $\left(r(t)=P_{f}(t)-P_{P V}(t)\right)$ and the measure of battery State Of Charge (SOC(t)). The measures used as feedback

Table 1. Set Point value for PID controllers

\begin{tabular}{cccc}
$\mathrm{r}(\mathrm{t})$ & $\mathrm{SOC}(\mathrm{t})$ & $r_{1}(t)$ & $r_{2}(t)$ \\
\hline$>0$ & $>25 \%$ & $r(t)$ & 0 \\
$>0$ & $<25 \%$ & 0 & 0 \\
$<0$ & $<85 \%$ & 0 & $|r(t)|$ \\
$<0$ & $>85 \%$ & 0 & 0 \\
\hline
\end{tabular}

by the two controllers are the power (W) absorbed and fed to the power line $\left(P_{C H}(t)\right.$ and $\left.P_{I N V}(t)\right)$, while the output of both PID controllers $\left(m_{1}(t)\right.$ and $\left.m_{2}(t)\right)$ is a current $(\mathrm{mA})$, necessary to drive the inverter and battery charger. The PID sampling time is $100 \mathrm{~ms}$, while, as descibed on the following, the supervisor acts every $60 \mathrm{~s}$, computing the correct setpoint and the appropriates PID improvements.

\section{IMPROVED PID CONTROLLER}

The PID function can be expressed as follows,

$$
m(t)=k_{p} e(t)+k_{i} e_{i}(t)+k_{d} e_{d}(t)
$$

where $e(t)$ is the error function, $k_{p}$ is the proportional gain, $k_{i}$ is the integration gain and $k_{d}$ is the derivative gain. $e(t)$, the difference between the desired input value and the actual output, will be sent to the PID controller, and the controller calculates both the derivative $e_{d}(t)$ and the integral $e_{i}(t)$ of this error function to control the system.

The proportional controller, the first term in (1), is the proportional action, which provides an overall control action and can reduce the error between the desired input value, and the actual output, but cannot fully eliminate this error. The integral controller, the second term in (1), has the effect of eliminating the steady-state error. When the error lasts, it can gradually enhance the control strength. But it may cause the transient response worsening. The derivative controller, the third term, has the effect of increasing the stability of the system, improving the transient response through high-frequency compensation by a differentiator. The incremental PID algorithm is an improved vision of the PID algorithm. Unlike the above PID controller, it is suitable to eliminate the error accumulation influence on the system, and its output takes the incremental form of (1) and is defined as:

$\Delta m(K)=k_{p} \Delta e(K)+k_{i} e(K)+k_{d}[\Delta e(K)-\Delta e(K-1)](2)$

where $\Delta e(K)$ can be expressed as follows, $\Delta e(K)=$ $e(K)-e(K-1)$ In the following the main features introduced to improve PID performances are described. Notice that the reference to each PID controller changes every 60 seconds according to the rules in table 1 .

\subsection{Adding Dead Zone}

When system comes into steady state, the deviation is very little. If deviation $e(K)$ fluctuates in a little range, the kernel controller will output a little control variable after computing the tiny deviation. As a result, the output controlled value, in a little range, incessantly changes 
its direction, frequently acts, causes vibration, which not only affect the output controller, but also disadvantage to battery which is charging. To solve this problem, we can apply the PID algorithm with adding dead zones, the control expression is:

$$
e(K)=\left(\begin{array}{cl}
0 & \text { if }|e(K)|<e_{0} \\
e(K) & \text { if }|e(K)|>e_{0}
\end{array}\right)
$$

where $e(K)$ is location tracking deviation, and $e_{0}$ is a adjustable variable. The specific values of $e_{0}$ is determined by the supervisor every 60 seconds based on the sampling precision of the devices and by the percentage error between the RBF network prediction and PV production.

\subsection{Speed-Change Integral}

In PID control algorithm, the role of the integral part is to eliminate static error and improve control accuracy. The requirement under the system of integral is that integral role should be reduced or even nothing when deviation is large, and should be strengthened when deviation is little. In the PID algorithm, the integral coefficient will produce bigger overshoot or even integral saturation when taken large value and can not eliminate the static error when taken small value. To solve this problem in the battery charge control system, this article suggested that change the cumulative rate of integral term on the basis of the measured values and the setting value of deviation, and achieved remarkable results. The idea is to modify the integral expression in $(2)$ to $f(e(K)) k_{i} e(K)$, where:

$$
f(e(K))=\left(\begin{array}{cc}
0 & \text { if }|e(K)|>e_{M A X} \\
\frac{e_{M A X}-e(K)}{e_{M A X}} & \text { if }|e(K)| \leq e_{M A X}
\end{array}\right)
$$

Notice that $e_{M A X}$ is the absolute value of the initial tracking deviation, computed by the supervisor every 60 seconds, when a new setpoint is defined.

\subsection{Anti-Integration Saturation}

The so called integral saturation is that, the output of the PID controller, as the accumulation of the integrals influence, makes its actuator reached the limiting position (maximum or minimum), but the output $\mathrm{m}(\mathrm{K})$ calculated by the controller is increasing still, and the value opening is not be further increased, it is the time that the controlled output from the controller is out of the normal range and fall in saturation region. If the reversal value happened in the system, $\mathrm{m}(\mathrm{K})$ will quit from the saturation region, the deeper it be in the saturation, the longer of time it takes to quit. During the time, actuator is still in the limiting position, and cannot act immediately as the reversal values change, at this time the system makes the degradation of properties as out of control. This phenomenon is called integral saturation. The idea of anti-integral saturation is that judge the controlled output of last time is out of the range or not when calculate $\mathrm{m}(\mathrm{K})$, if $m(K-1)>U_{\max }$, only add the negative deviation; if $m(K-1)<U_{m i n}$, only add the positive deviation. This algorithm can prevent the control variable in the saturation region for a long time and make possible the following of the time varying setpoint (one of the main features of this work) without having longer transient.

\section{PV PRODUCTION FORECAST}

Our forecasting scheme, based on RBF Neural Network, is used to derive predictions of solar irradiation based on forecasts up to 48 hours ahead provided by meteo websites and Energy Resources' database of measured solar irradiation. The irradiation forecast is used as input for a PV's model to obtain the forecast of the PV production. The irradiation is forecasted on the plant's panel plane and orientation. The RBF network algorithm proposed by Cavalletti et al. (2007), Corradini et al. (2003) and Chen et al. (1991) is used with the set of experimental data composed by the pairs $(x(n), y(n)), n=1,2, \ldots$ where $x(n)$ contains the whether forecast, the number of day of the year, the hour of the day, and $y(\cdot)$ is the solar irradiation. Sampling time is one hour and datasets have been also normalized, between 0 and 1 in order to have the same range. Tests are based on data acquired from August 2010 to July 2011 during PV plant standard working. In particular two different data sets have been considered; one is relative to the first 12 days of each month and is used for the training, while the second is relative to remaining days and is for the validation of the network. These data sets are acquired with a significative time lag between them in order to assure independent data sets. A sample of the results on training the network on the first data set is shown in Figure 2, while the behaviour of the network on the second set (validation) is shown in figure 3. The red-continuous line is the predicted irradiation, while the blue-dashed line is the measured one.

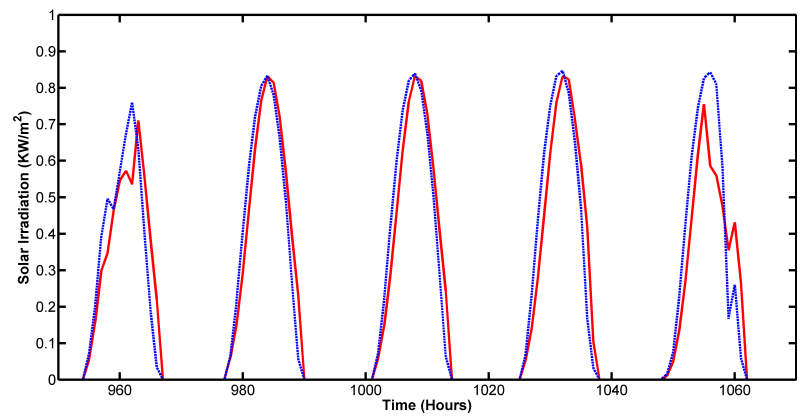

Fig. 2. Predicted (continuous red line) and Measured (blue dashed line) Irradiation for 5-9 May 2011

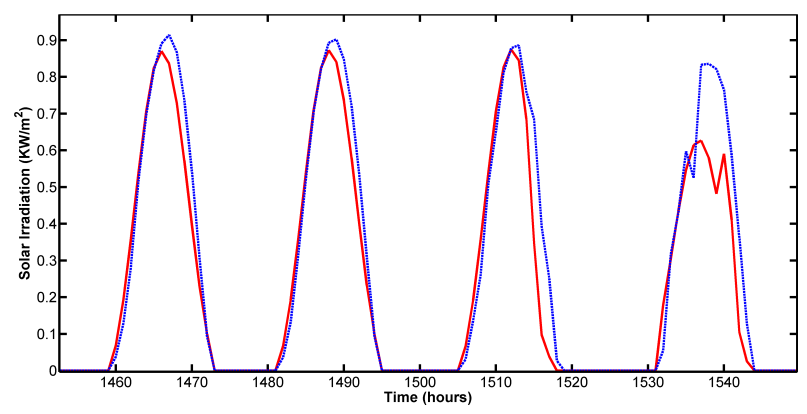

Fig. 3. Predicted (continuous red line) and Measured (blue dashed line) Irradiation for 20-23 May 2011

Using a simple PV plant model to estimate the power production, as described in Gonzlez-Longatt (2005), the hourly irradiation prediction can be used to generate the desired power reference. The input parameters of the PV 
model are the irradiation (forecasted with the RBFN) and the module temperature; an approximate expression for calculating the cell temperature is given by (Ross (1980)):

$$
T_{\text {cell }}=T_{\text {air }}+\frac{(N O C T-20) \operatorname{Irr}}{800}
$$

where $T_{\text {cell }}$ is the cell temperature, $T_{\text {air }}$ the air temperature provided by weather forecasts, Irr is the forecasted irradiation in $\left[\mathrm{W} / \mathrm{m}^{2}\right]$ and NOCT is the Nominal Operating Cell Temperature, a specif parameter for each kind of PV module (see Table 5).

\section{IMPLEMENTATION SETTING}

The proposed improved PID scheme has been implemented to schedule the power feeding of one of the PV plants of the italian company Energy Resouces, in Jesi (AN), Italy. The RBF Neural Network forecast has been implemented to predict energy production of the same plant. The experimental setup is shown in fig. 1 . It is a combination of hardware and software and includes nine solar inverters ( 8 of them connected to Renergies solar panel and the last to the battery pack), a lithium battery pack, a Battery Management System (BMS) and a software platform to develop forecast and control the feeding of produced power. All communication between software and hardware is done through TCP/IP protocol, using serial to TCP/IP conveters and connecting them to a server, in which are located the softwares to manage the whole system. For what regards the tuning of PID parameters has been chosen the Tyreus Luyben tecnique (see Tyreus et al. (1992)). Different tuning techniques, as described in Astrom et al. (1995) and Skogestad (2001), have been tested without significative improvements of the performance. The adopted solution (giving a different setpoint every $60 \mathrm{~s}$ ) is useful to limitate the cycles of charge/discharge (DOD) of the battery but at the same time introduces an error on the hourly energy provided to the power line of about $1 / 60$ of the hourly energy reference. It's possible to use this solution because the law proposal, discussed in section 1 admitted an error of maximum 10\% on the effective hourly energy provided. In this section, the plant structure, the experimental setup and the results are discussed.

\subsection{Experimental Setup}

Experimental tests have been performed on a PV plant composed by 8 strings of Renergies $220 \mathrm{P} / 220$ polysilicon panels (Renergies website (2011)). Each string is connected to a SMA Sunny Boy 1700IT solar inverter (SMA website (2011)). A lithium battery pack is composed by the series of two sub-module with 80 ThunderSky modules 40 Ah, a Battery Management System (BMS) and a battery charger for each module (Winston Battery website (2011)). A solar inverter (model SIAC soleil 10Kw) is connected to this pack (Siel website (2011)). The parameters of the system are listed in table $2,3,4$ and 5 .

\subsection{Experimental Results}

Figs. 4 and 5 show the performance of the improved supervised PID and a PID with the same parameters
Table 2. Technical Data Inverter Sunny Boy 1700IT

\begin{tabular}{cccc} 
Input Data & \multicolumn{3}{c}{ Output Data } \\
\hline Max DC Pow & $1850 \mathrm{~W}$ & Nominal AC Pow & $1550 \mathrm{~W}$ \\
Max Efficiency & 0.935 & Max AC Pow & $1.7 \mathrm{KW}$ \\
Max Current & $12.6 \mathrm{~A}$ & Max Current & $8.6 \mathrm{~A}$ \\
\hline
\end{tabular}

Table 3. Technical Data Inverter Siac Soleil 10

\begin{tabular}{cccc} 
Input Data & \multicolumn{3}{c}{ Output Data } \\
\hline Recom DC Pow & $8-12 \mathrm{KW}$ & Max AC Volt & $400 \mathrm{~V}$ \\
DC Volt MPPT & $139-320 \mathrm{~V}$ & Max AC Pow & $9 \mathrm{KW}$ \\
Max Current & $29 \mathrm{~A}$ & Max Efficiency & 0.93 \\
\hline
\end{tabular}

Table 4. Battery Pack Specifications

\begin{tabular}{cccc}
\hline Max Mod V & $300 \mathrm{~V}$ & Max Charge I & $25 \mathrm{~A}$ \\
Min Mod V & $200 \mathrm{~V}$ & Max Discharge I & $40 \mathrm{~A}$ \\
Mod Capacity & $10 \mathrm{Ah}$ & Life Cycles (80\% DOD) & 3000 \\
\hline
\end{tabular}

Table 5. Technical Data Renergies 220P/220

\begin{tabular}{cccc}
\hline Max Pow & $220 \mathrm{~W}$ & Coeff Pmax $/ \mathrm{T}$ & $-0.43 \% /{ }^{\circ} \mathrm{C}$ \\
Isc & $8.5 \mathrm{~A}$ & Coeff Isc $/ \mathrm{T}$ & $4.00 \mathrm{~mA} /{ }^{\circ} \mathrm{C}$ \\
Voc & $36.6 \mathrm{~V}$ & Coeff Voc $/ \mathrm{T}$ & $-126 \mathrm{mV} /{ }^{\circ} \mathrm{C}$ \\
Max Pow V & $28.11 \mathrm{~V}$ & Max Pow I & $7.87 \mathrm{~A}$ \\
\hline
\end{tabular}

but without improvements and supervision. In particular, during the transient caused by changes in the references, the improved PID (Figs. 4 and 5 - dashed black line) show a better tracking performance, moreover on the second step setpoint change, with respect to the standard PID (Figs. 4 and 5 - blue line). A sample of the behaviour of the discharge PID (related to the inverter) is shown in figure 4 while the charge PID is shown in figure 5 . The setpoint, computed by supervisor has two step variations (at time 0 and 120), and it's represented with red dotted line. Notice that, before the first step setpoint change (at time 0 ) the inverter and battery charger are not generating (or absorbing) power.

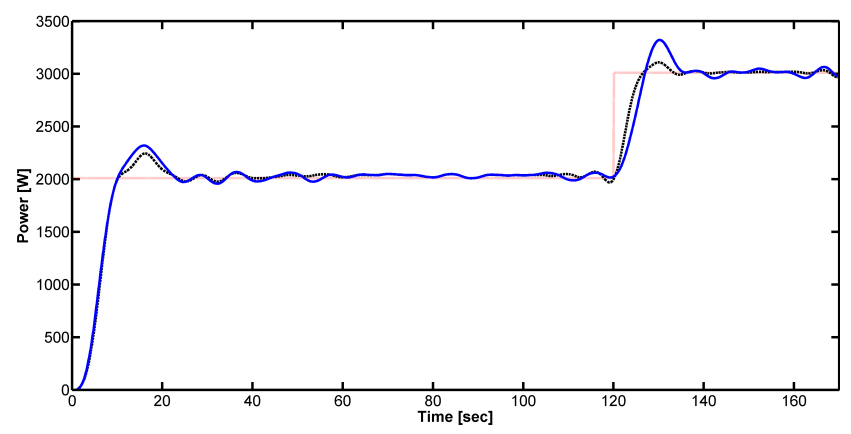

Fig. 4. Performance of the Discharge PID controller

Specifications on set-points following include requirements on rise time, settling time and overshoot for step changes in setpoint. With reference to the first setpoint step change, these parameters are reported in the Table 6 for the improved PID controllers and in 7 for the standard one. Another performance index, the Integral of absolute error (IAE, calculated for the first step) is presented.

A sample of the system's behaviour is shown in figure 6 a. Datas refers to the period 24-27 august, during standard working of the system. Production forecasted is represented with red continuous line, while power produced by the PV plant with blue dotted line. In figure $6 \mathrm{~b}$ it's 


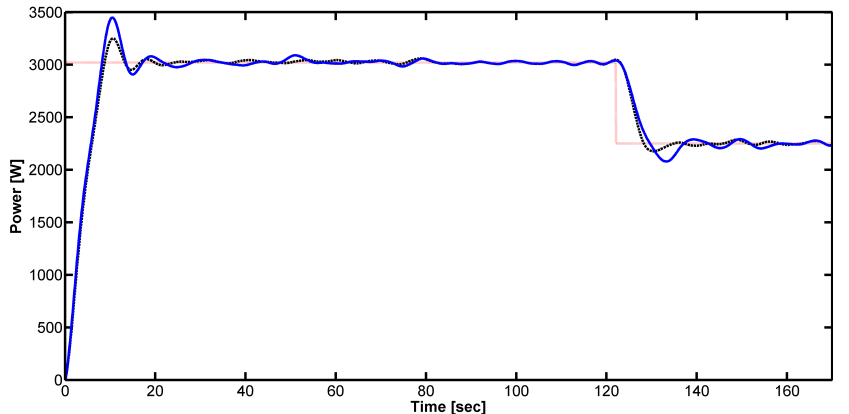

Fig. 5. Performance of the Charge PID controller

Table 6. Performance of improved PID controllers

\begin{tabular}{ccccc}
\hline & Rise Time & Settling Time & Overshoot & IAE \\
Discharge & $4.6 \mathrm{~s}$ & $8.2 \mathrm{~s}$ & $7.2 \%$ & 1308 \\
Charge & $3.2 \mathrm{~s}$ & $7.1 \mathrm{~s}$ & $3.7 \%$ & 1544 \\
\hline
\end{tabular}

Table 7. Performance of standard PID controllers

\begin{tabular}{ccccc}
\hline & Rise Time & Settling Time & Overshoot & IAE \\
Discharge & $4.6 \mathrm{~s}$ & $11.2 \mathrm{~s}$ & $13.2 \%$ & 1450 \\
Charge & $3.2 \mathrm{~s}$ & $11.1 \mathrm{~s}$ & $9.7 \%$ & 1636 \\
\hline
\end{tabular}

reported the SOC of the battery during the same period. Notice that night time is not reported in figure (datas from 8.30 p.m. to 6.30 a.m.).
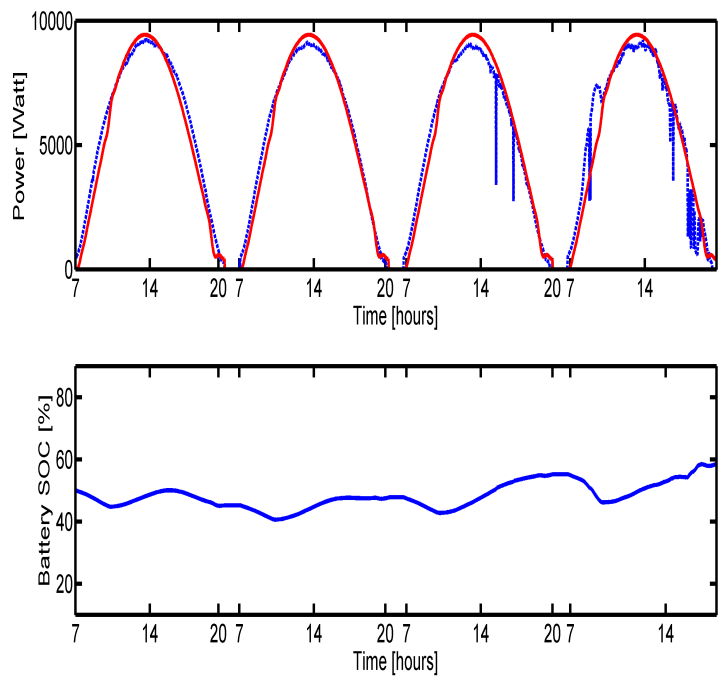

Fig. 6. a) Production forecasted and measured for 24-27 August, b) Battery SOC during the same period

The hourly Root Mean Square of the Error (RMSE) between desired power reference (computed by RBF Neural Network and the PV model, constant in an hour) and total balance of power feeding the power line (measured each $100 \mathrm{~ms}$ ) is used as a performance indicator. RMSE\% for an hour (in which $P_{f}$ is a constant) is calculated as follows:

$$
\begin{gathered}
E(K)=P_{f}-P_{P V}(K)-P_{I N V}(K)+P_{C H}(K) \\
R M S E \%=\frac{\sqrt{\sum_{K=1}^{n} E(K)^{2} / n}}{P_{f}}
\end{gathered}
$$

While the percentage error is:

$$
E \%=\frac{\sum_{K=1}^{n} E(K) / n}{P_{f}}
$$

Datas from 1st July to 10th September show an RMSE\% of $7.9 \%$, and an average $\mathrm{E} \%$ of $5.7 \%$. Those results make possible the schedulling of PV produced energy with an hourly error less tha $10 \%$ in 67 of the 71 days considered

\section{CONCLUDING REMARKS}

We have presented an improved PID controller with a supervisor to provide the power line with a scheduled profile from PV plant's production using a simple system composed by lithium batteries and a solar inverter. A Neural Network based approach is used to derive hourly irradiance forecast for the city of Jesi (AN), Italy on the panel's plane from daily wheather forecasts as a basis to predict $\mathrm{PV}$ power output. The comparison between the presented controller and a standard PID with the same parameters shows an improvement of settling time, a better overshoot and IAE. The indexes used to evaluate the performance of the power scheduling are the hourly percentage RMSE of $7.9 \%$ and $\mathrm{E} \%$ of $5.7 \%$. The main factor that reduces accuracy of control is, of course, a sudden changing of the weather, that implies a sudden variation on the $\mathrm{PV}$ production, moreover on what regards cloudness or cloudless of a sunny or a cloudy day. An optimization of the capacity of the batteries and a new control strategy is under development. Furthermore we are extending the proposed forecasting approach to a bigger region (the entire Italy) for all panels' orientation and tilting.

\section{ACKNOWLEDGEMENTS}

Special thanks to Energy Respurces SPA, an italian company of Jesi $(\mathrm{AN})$, leader in renewable energies' market

\section{REFERENCES}

M. Cavalletti, G. Ippoliti, S. Longhi, Lyapunov-based switching control using neural networks for a remotely operated vehicle, Int. J. of Contr. 80 (7) (2007) 10771091.

D'Amico, A., Ippoliti, G. , Longhi, S., A multiple models approach for adaptation and learning in mobile robots control, Journal of Intelligent and Robotic Systems 47(1), 3-31, 2006.

R.P. Copeland, K.S. Rattan, A fuzzy logic supervisor for PID control of unknown systems, IEEE International Symposium on Intelligent Control, pp.22-26, 16-18 Aug 1994.

Antonini, P., Ippoliti, G. , Longhi, S. , Learning control of mobile robots using a multiprocessor system, Control Engineering Practice 14(11), 1279-1295, 2006.

Z. Liu and C.P., Ji, Application of Improved PID Algorithm in the Battery Charge Control System, International conference on Challenges in Environmental Science and Computer Engineering (CESCE), vol.1, no., pp.190-192, 6-7 March 2010. 
S. Chen, C. F. N. Cowan, P. M. Grant, Orthogonal least squares learning algorithm for radial basis function networks, IEEE Trans. Neural Networks 2 (2) (1991) 302309.

M. Corradini, G. Ippoliti, S. Longhi, Neural networks based control of mobile robots: development and experimental validation, J. Robot. Syst. 20 (10) (2003) 587-600.

R. Sehab, Fuzzy PID Supervision for a Nonlinear, System: Design and Implementation, Fuzzy Information Processing Society, NAFIPS '07, pp.36-41, 24-27 June 2007.

S. Skogestad, Probably the best simple PID tuning rules in the world, AIChE Annual Meeting, Reno, Nevada, November 2001.

K. Astrom, T. Hagglund, PID Controllers: Theory, Design, and Tuning, ISA: The Instrumentation, Systems, and Automation Society, January 1995.

B. Tyreus, W. Luyben, Tuning PI controllers for integrator/dead time processes, Ind. Eng. Chem. Res. (1992), 31 (11), pp 26252628.

G. Ippoliti, L. Ietto and S. Longhi, Improved setpoints tracking of remotely operated underwater vehicles through a supervised PID control scheme, Journal of Marine Engineering and Technology, Volume 2005, Number 7, December 2005 , pp. 3-9(7).

K. Strunz and E.K. Brock, Hybrid Plant of Renewable Stochastic Source and Multi-Level Storage for EmissionFree Deterministic Power Generation, Quality and Security of Electric Power Delivery Systems CIGRE/IEEE PES International Symposium , vol., no., pp. 214- 218, 8-10 Oct. 2003.

Lange, M. ,A mature market? The history of short term prediction services POW'WOW Best Practice Workshop, Delft, The Netherland, 2006.

Lange, M. , Focken, U. ,Physical approach to short term wind power prediction, New York: Springer, 2005.

Li, J., Song, A.G. ,Comparison of clear-day solar radiation model in Beijing to ASHRAE model, Journal of Capital Normal University, vol. 19, No. 1, pp 35-38, 1998.

Ren, M.J. , Wright, J.A. ,Adaptative diurnal prediction of ambient dry-bulb temperaature and solar radiation,HVAC and Research, Vol. 8, No. 4, 2002, pp. 383401.

Dorvlo, A.S.S. , Jervase, J.A., Ali, A.L. ,Solar radiation estimation using artificial neural networks, Applied Energy,71: 307-319, 2002.

Gonzlez-Longatt, F.M. ,Model of Photovoltaic Module in Matlab,II CIBELEC ,2005.

Lorenz, E., Hurka, J., Heinemann, D., Beyer, H.G., Irradiance Forecasting for the Power Prediction of GridConnected Photovoltaic Systems, IEEE Journal of Selected Topics in Applied Earth Observations and Remote Sensing, vol.2, no.1, pp.2-10, March 2009.

L. Ljung, System identification, theory for the user, Information and System Sciences Series, Prentice Hall PTR, New Jersey, USA, 1999.

Ross, R.G., Flat-Plate Photovoltaic Array Design Optimization, Conference Record, 14th IEEE Photovoltaic Specialists Conference, San Diego, USA , pp. 1126-1132, 1980.

Renergies Italia, [Online] Available: http://www.renergiesitalia.it/

SMA Solar Technology, [Online] Available: http://www.sma-italia.com/it/prodotti/inverter- solari/sunny-boy.html

Siel Energy and Safety, [Online] Available: http://www.sielups.com/INVERTER/Fotovoltaico/SIACSoleil-10-TL.aspx

Winston Battery, [Online] Available: http://en.winstonbattery.com/ 